\title{
ABSOLUTE CONVERGENCE OF CONTINUED FRACTIONS ${ }^{1}$
}

RALPH E. LANE

1. Introduction. Consider the continued fraction

$$
f_{1}+\frac{a_{1}}{b_{1}-\frac{a_{2}}{b_{2}-\frac{a_{3}}{b_{3}-\cdots}},}
$$

where $f_{1}$ is a number, $\left\{a_{1}, a_{2}, a_{3}, \cdots\right\}$ is a sequence of nonzero numbers, and $\left\{b_{1}, b_{2}, b_{3}, \cdots\right\}$ is a sequence of numbers. We obtain conditions necessary and sufficient for (1.1) to converge absolutely, and we indicate their relationship to older sufficient conditions. We find a new characterization of positive definite continued fractions, whose importance is emphasized by the fact (Theorem 4.2) that if (1.1) converges, then there is a positive definite continued fraction which is a contraction of (1.1). We also obtain new sufficient conditions for absolute convergence of positive definite continued fractions.

2. Continued fractions and sequences of linear fractional transformations. In this paper, a subscript $p$ denotes a positive integer. By the generator of (1.1) we mean the sequence $\left\{t_{1}(u), t_{2}(u)\right.$, $\left.t_{3}(u), \cdots\right\}$ of linear fractional transformations such that $t_{1}(u)$ $=f_{1}+a_{1} /\left(b_{1}-u\right)$ and $t_{p+1}(u)=t_{p}\left[a_{p+1} /\left(b_{p+1}-u\right)\right]$ for $p \geqq 1$. We denote this sequence by $t(u)$.

REMARK 2.1. For a sequence $s(u)$ of linear fractional transformations to be the generator of a continued fraction, it is necessary and sufficient that $s_{1}(\infty) \neq \infty$ and $s_{p}(0)=s_{p+1}(\infty)$ for $p \geqq 1$.

By the sequence of approximants of (1.1) we mean the sequence $\left\{f_{1}, f_{2}, f_{3}, \cdots\right\}$ such that $f_{p}=t_{p}(\infty)$ for $p \geqq 1$. We denote this sequence by $f$.

REMARK 2.2. For a sequence $x$ of points in the complex plane to be the sequence of approximants of a continued fraction with nonzero partial numerators, it is necessary and sufficient that $x_{1} \neq \infty$ and $x_{p}$ $\neq x_{p+1}$ for $p \geqq 1$.

Presented to the Society, August 30, 1949; received by the editors January 10 , 1952.

${ }^{1}$ This paper is based on a thesis written under the direction of Professor H. S. Wall of the Department of Pure Mathematics, The University of Texas. 
If $f$ has the property that there exists a positive integer $n$ such that (1) the sequence $\left\{f_{n}, f_{n+1}, f_{n+2}, \cdots\right\}$ is bounded and (2) either $n=1$ or $f_{n-1}=\infty$, then by $B_{f}$ we mean the set of all sequences $R$ such that for $p \geqq 1$

(i) $R_{p}$ is a circle plus its interior,

(ii) $R_{p} \supset R_{p+1}$, and

(iii) $f_{p}$ is in $R_{p}$ if $p \geqq n$.

Theorem 2.1. If $f$ is bounded, then for $R$ to be a member of $B_{f}$ it is necessary and sufficient that

(i) $R_{1}$ is a circle plus its interior,

(ii) if $p \geqq 1$, then $t_{p}^{-1}\left(R_{p}\right)$ is a closed half-plane or a circle plus its exterior, and

(iii) if $p \geqq 1$, then $t_{p}^{-1}\left(R_{p}\right) \supset t_{p}^{-1}\left(R_{p+1}\right)$.

Moreover, if $R$ is a sequence in $B_{f}$, and if $p \geqq 1$, then $t_{p}^{-1}\left(R_{p}\right)$ is a closed half-plane if $f_{p}$ is a boundary point of $R_{p}$, or is a circle plus its exterior if $f_{p}$ is an interior point of $R_{p}$.

Proof. The theorem is a direct consequence of the definitions of $f$, $t(u)$, and $B_{f}$.

We denote by $h$ the sequence $\left\{h_{1}, h_{2}, h_{3}, \cdots\right\}$ of points in the complex plane such that if $p \geqq 1$ then $h_{p}=t_{p}^{-1}(\infty)$. From the relations $t_{1}\left(b_{1}\right)=\infty$ and $t_{p+1}(u)=t_{p}\left[a_{p+1} /\left(b_{p+1}-u\right)\right]$, it follows that

$$
h_{1}=b_{1} \quad \text { and } \quad h_{p+1}=b_{p+1}-a_{p+1} / h_{p} \quad \text { for } p \geqq 1 .
$$

If $p \geqq 1$, then $t_{p}(\infty)=f_{p}, t_{p}(0)=f_{p+1}$, and $t_{p+1}\left(b_{p+1}\right)=f_{p}$; so that

$$
\begin{aligned}
f_{p} & =\infty \text { if and only if } h_{p}=\infty, \\
f_{p+1} & =\infty \text { if and only if } h_{p}=0, \text { and } \\
f_{p} & =\infty \text { if and only if } h_{p+1}=b_{p+1} .
\end{aligned}
$$

If $p \geqq 1$, and if $f_{p} \neq \infty$ and $f_{p+1} \neq \infty$, then

$$
t_{p}(u)=f_{p}+\frac{h_{p}\left(f_{p+1}-f_{p}\right)}{h_{p}-u} .
$$

If $p \geqq 1$, and if $f_{p} \neq \infty, f_{p+1} \neq \infty$, and $f_{p+2} \neq \infty$, then

$$
\frac{f_{p+1}-f_{p+2}}{f_{p}-f_{p+1}}=\frac{b_{p+1}-h_{p+1}}{h_{p+1}}=\frac{a_{p+1}}{h_{p} h_{p+1}} .
$$

3. Conditions necessary and sufficient for absolute convergence. If $x$ is a sequence of points in the complex plane, the statement that $x$ converges absolutely means that there exists a positive integer $n$ 
such that (1) if $p \geqq n$, then $x_{p} \neq \infty$ and (2) $\sum_{p=n}^{\infty}\left|x_{p}-x_{p+1}\right|$ converges. The statement that a continued fraction converges absolutely means that its sequence of approximants converges absolutely.

THEOREM 3.1. For (1.1) to converge absolutely, it is necessary and sufficient that there exist a positive integer $n$, a sequence $s$ of numbers, and a sequence $q$ of numbers such that

(i) $s_{p}>0$ and $q_{p} \neq 0$ for $p \geqq n$, and $\sum_{p=n}^{\infty} s_{p}$ converges,

(ii) there is a sequence $R$ in $B_{f}$ such that if $p \geqq n$, then $t_{p}^{-1}\left(R_{p}\right)$ is the region defined by the inequality $s_{p}|u| \leqq\left|u-q_{p}\right|$, and

(iii) there is a sequence $R^{\prime}$ in $B_{f}$ such that if $p \geqq n$, then $q_{p}$ is in $t_{p}^{-1}\left(R_{p}^{\prime}\right)$.

Proof. A. Suppose that there exist such an integer $n$ and such sequences $s$ and $q$. Let $m$ denote an integer such that if $p \geqq m$, then $p \geqq n$ and $f_{p}$ is in $R_{p}$. Now $R_{p}$ is a circle plus its interior, $\infty$ is not in $R_{p}$, and $h_{p}=t_{p}^{-1}(\infty)$ is not in $t_{p}^{-1}\left(R_{p}\right)$; hence if $p \geqq m$, then $s_{p}\left|h_{p}\right|$ $>\left|h_{p}-q_{p}\right|$, or $s_{p}>\left|\left(h_{p}-q_{p}\right) / h_{p}\right|$. Moreover, if $p \geqq m$, then by (2.4) and (2.5),

$$
\left|\left(f_{p+1}-f_{p}\right) /\left[t_{p}\left(q_{p}\right)-f_{p}\right]\right|=\left|\left(h_{p}-q_{p}\right) / h_{p}\right|<s_{p} .
$$

By hypothesis, $f_{p}$ is in $R_{m}$ and $t_{p}\left(q_{p}\right)$ is in $R_{m}^{\prime}$, and consequently there exists a number $M$ such that if $p \geqq m$, then $t_{p}\left(q_{p}\right)-f_{p}<M$, so that $\left|f_{p+1}-f_{p}\right|<M s_{p}$. Since $\sum_{p=n}^{\infty} s_{p}$ converges, (1.1) converges absolutely.

B. Suppose that (1.1) converges absolutely. Let $n$ denote the positive integer such that if $p \geqq n$, then $f_{p} \neq \infty$ and such that either $n=1$ or $f_{n-1}=\infty$. Let $R_{n}$ be a circle plus its interior, with radius $r$ and center $c$ such that if $p \geqq n$, then $3 r>4\left|f_{p}-c\right|>2 r$. Let $R_{n}^{\prime}$ be a circle plus its interior with radius $r^{\prime}$ and center $c$, such that $R_{n}^{\prime} \supset R_{n}$ and such that if $p \geqq n$, then the inversion of $f_{p}$ in the boundary of $R_{n}$ is in $R_{n}^{\prime}$. For $p \geqq 1$, let $R_{p}=R_{n}$ and $R_{p}^{\prime}=R_{n}^{\prime}$. Then $R$ is in $B_{f}$ and $R^{\prime}$ is in $B_{f}$.

For $p \geqq n$, let $t_{p}\left(q_{p}\right)$ be the inversion of $f_{p+1}$ in the boundary of $R_{p}$. By construction, $t_{p}\left(q_{p}\right)$ is in $R_{p}^{\prime}$, so that $q_{p}$ is in $t_{p}^{-1}\left(R_{p}^{\prime}\right)$. Moreover, if $p \geqq n$, then there exists a positive number $s_{p}^{\prime}$ such that $R_{p}$ is the region defined by $s_{p}^{\prime}\left|u-f_{p+1}\right| \leqq\left|u-t_{p}\left(q_{p}\right)\right|$; and since $3 r>4\left|f_{p+1}-c\right|>2 r$, there exist positive numbers $D$ and $s^{\prime}$ such that $\left|t_{p}\left(q_{p}\right)-f_{p}\right| \geqq D$ and $s_{p}^{\prime} \leqq s^{\prime}$ for $p \geqq n$. By $(2.5), t_{p}^{-1}\left(R_{p}\right)$ is the region defined by $s_{p}|u| \leqq\left|u-q_{p}\right|$, where $s_{p}=s_{p}^{\prime}\left|\left(f_{p+1}-f_{p}\right) /\left[t_{p}\left(q_{p}\right)-f_{p}\right]\right|$ $<\left|f_{p+1}-f_{p}\right| s^{\prime} / D$. Hence $\sum_{p=n}^{\infty} s_{p}$ converges. This completes the proof.

LEMMA 3.2a. If $s$ is a sequence of positive numbers, then for $\sum_{p=1}^{\infty} s_{p}$ to converge it is necessary and sufficient that there exist a sequence $d$ of 
positive numbers such that for $p \geqq 1$

$$
\frac{s_{p+1}}{s_{p}} \leqq \frac{d_{p}}{1+d_{p+1}} .
$$

Proof. Suppose that $d$ is such a sequence. If $p \geqq 1$, then $s_{p+1}$ $+s_{p+1} d_{p+1} \leqq s_{p} d_{p}$; and by induction, if $n$ is an integer greater than $p$, then $\sum_{k=p+1}^{n} s_{k}+s_{n} d_{n} \leqq s_{p} d_{p}$, so that $\sum_{k=1}^{n} s_{k}<\sum_{k=1}^{p} s_{k}+s_{p} d_{p}$. Hence $\sum_{k=1}^{\infty} s_{k}$ converges.

Suppose that $\sum_{k=1}^{\infty} s_{k}$ converges. Let $r$ be a sequence of non-negative real numbers such that $\sum_{k=1}^{\infty} r_{k}$ converges, and for $p \geqq 1$, let $d_{p}$ be the positive number such that $s_{p} d_{p}=\sum_{k=p+1}^{\infty}\left(r_{k}+s_{k}\right)$. Then $s_{p} d_{p}$ $=r_{p+1}+s_{p+1}+s_{p+1} d_{p+1} \geqq s_{p+1}+s_{p+1} d_{p+1}$, so that $s_{p+1} / s_{p} \leqq d_{p} /\left(1+d_{p+1}\right)$. This completes the proof.

REMARK 3.1. From the above proof it follows that if in Lemma 3.2a the statement $s_{p+1} / s_{p} \leqq d_{p} /\left(1+d_{p+1}\right)$ is replaced by either of the statements

$$
\frac{s_{p+1}}{s_{p}}<\frac{d_{p}}{1+d_{p+1}}, \quad \frac{s_{p+1}}{s_{p}}=\frac{d_{p}}{1+d_{p+1}},
$$

then the resulting lemma is true.

EXAMPLE 3.1. Let $a>-1, b>a+1$, and $d_{p}=(a+p) /(b-a-1)$ for $p \geqq 1$. By Lemma 3.2a, the series

$$
1+\frac{a+1}{b+1}+\frac{(a+1)(a+2)}{(b+1)(b+2)}+\cdots
$$

converges.

LEMMA 3.2b. For $f$ to converge absolutely, it is necessary and sufficient that there exist a positive integer $n$ and a sequence $d$ of positive numbers such that, for $p \geqq n$,

(i) $d_{p}>1+d_{p+1}$ if $f_{p+1}=\infty$ or if $f_{p}=f_{p+2}=\infty$ and

(ii) $d_{p}\left|f_{p}-f_{p+1}\right|>\left(1+d_{p+1}\right)\left|f_{p+1}-f_{p+2}\right|$ if
(a) $f_{p+1} \neq \infty$ and
(b) $f_{p} \neq \infty$ or $f_{p+2} \neq \infty$.

Proof. If $f$ converges absolutely, then there exists a positive integer $n$ such that $f_{p} \neq \infty$ if $p \geqq n$; and by Remark 3.1 there exists a sequence $d$ of positive numbers such that (ii) holds for $p \geqq n$.

Suppose that there exist a positive integer $n$ and a sequence $d$ of positive numbers such that (3.2) holds for $p \geqq n$. We first show that if $p \geqq n+d_{n}$, then $f_{p} \neq \infty$. Suppose that $m$ is an integer, that $m \geqq n$ $+d_{n}$, and that $f_{m}=\infty$. Then for $p=m-1$, the relation (i) holds by hypothesis, and $d_{m-1}>1+d_{m}>1$. Since $f_{m}=\infty$, it follows (Remark 
2.2) that $f_{m-1} \neq \infty$. If $f_{m-2} \neq \infty$, therefore, (ii) must hold for $p=m-2$; but this is impossible, since $f_{m}=\infty$. Hence $f_{m-2}=\infty$, and (i) holds for $p=m-2$, so that $d_{m-2}>1+d_{m-1}>2$. If $m>n+2$, then (i) must hold for $p=m-3$, and $d_{m-3}>3$. If $m>n+3$, then $f_{m-4}=\infty$ and $d_{m-4}>4$. By induction, $d_{n}>m-n$, so that $m<n+d_{n}$. Hence the assumption that $f_{m}=\infty$ is false; and if $p \geqq n+d_{n}$, then $f_{p} \neq \infty$. By Lemma 3.2a, $f$ converges absolutely. This completes the proof.

THEOREM 3.2. For (1.1) to converge absolutely, it is necessary and sufficient that there exist a positive integer $n$ and a sequence $d$ of positive numbers such that, for $p \geqq n$,

(i) if $b_{p+1}=0$, then $d_{p}>1+d_{p+1}$, and

(ii) if $b_{p+1} \neq 0$ and if $t_{p+1}^{-1}\left(K_{p+1}\right)$ is the region defined by $d_{p}|u|$ $\leqq\left(1+d_{p+1}\right)\left|u-b_{p+1}\right|$, then $K_{p+1}$ is a circle plus its interior.

Proof. The conditions (3.2) of Lemma $3.2 \mathrm{~b}$ can be written

(a) $d_{p}>1+d_{p+1}$ if $f_{p}=f_{p+2}$,

(b) $d_{p}>1+d_{p+1}$ if $f_{p} \neq f_{p+2}$ and $f_{p+1}=\infty$,

(c) $d_{p}\left|f_{p}-f_{p+1}\right|>\left(1+d_{p+1}\right)\left|f_{p+1}-f_{p+2}\right|$ if $f_{p} \neq f_{p+2}$ and $f_{p+1} \neq \infty$.

Since $f_{p}=t_{p+1}\left(b_{p+1}\right), f_{p+2}=t_{p+1}(0)$, and $\infty=t_{p+1}\left(h_{p+1}\right)$, the first two of these conditions can be written

(a') $d_{p}>1+d_{p+1}$ if $b_{p+1}=0$,

(b') $d_{p}>1+d_{p+1}$ if $b_{p+1} \neq 0$ and $h_{p+1}=\infty$;

as for the third, where $b_{p+1} \neq 0$ and $h_{p+1} \neq \infty$, similar consideration of the two cases (1) $h_{p+1}=0$ and (2) $h_{p+1}=b_{p+1}$, and use of (2.6) for the case (3) $h_{p+1} \neq 0, h_{p+1} \neq b_{p+1}$, shows that (c) may be written

(c') $d_{p}\left|h_{p+1}\right|>\left(1+d_{p+1}\right)\left|h_{p+1}-b_{p+1}\right|$ if $b_{p+1} \neq 0$ and $h_{p+1} \neq \infty$.

Now if $t_{p+1}^{-1}\left(K_{p+1}\right)$ is defined by $d_{p}|u| \leqq\left(1+d_{p+1}\right)\left|u-b_{p+1}\right|$, where $d_{p}>0, d_{p+1}>0$, and $b_{p+1} \neq 0$, then for $K_{p+1}$ to be a circle plus its interior, it is necessary and sufficient that the point $h_{p+1}=t_{p+1}^{-1}(\infty)$ be exterior to $t_{p+1}^{-1}\left(K_{p+1}\right)$. Hence the conditions $\left(\mathrm{a}^{\prime}\right),\left(\mathrm{b}^{\prime}\right)$, and $\left(\mathrm{c}^{\prime}\right)$ are equivalent to (3.3), and the theorem now follows from Lemma 3.2b. This completes the proof.

REMARK 3.2. If $a_{1}=1$ and $b_{p}=1$ and $a_{p+1}=-c_{p}$ for $p \geqq 1$, then (1.1) is the continued fraction

$$
\frac{1}{1+\frac{c_{1}}{1+\frac{c_{2}}{1+\cdots}}},
$$


where $c$ is a sequence of nonzero numbers. If, in the notation of Theorem 3.2, $r_{p}=d_{p} /\left(1+d_{p+1}\right)$, then $t_{p+1}^{-1}\left(K_{p+1}\right)$ is defined by the inequality $r_{p}|u| \leqq|u-1|$. The condition $t_{p}^{-1}\left(K_{p}\right) \supset t_{p}^{-1}\left(K_{p+1}\right)$ gives the inequalities $(5.5)$, p. 376 , of Lane and Wall $[1]^{2}$ for $p \geqq 1$. The condition $t_{p}^{-1}\left(K_{p-1}\right) \supset t_{p}^{-1}\left(K_{p-1 p+1}\right)$ gives, for $p \geqq 2$, the inequalities (13.) of Scott and Wall [2].

4. A characterization of positive definite continued fractions. The continued fraction (1.1) is said to be positive definite ${ }^{3}$ if

(i) $I\left(b_{1}\right)>0$ and $I\left(b_{p}\right) \geqq 0$ for $p>1$, and

(ii) there exists a sequence $g$ of numbers such that $0<g_{1} \leqq 1$ and, for $p \geqq 1,0 \leqq g_{p+1} \leqq 1$ and

$$
\left|a_{p+1}\right|-R\left(a_{p+1}\right) \leqq 2 I\left(b_{p}\right) I\left(b_{p+1}\right)\left(1-g_{p}\right) g_{p+1} \text {. }
$$

If $F$ is a continued fraction, the statement that $F$ is equivalent to (1.1) means that the sequence of approximants of $F$ is the sequence of approximants of (1.1).

REMARK 4.1. If $F$ is a continued fraction, and if $t^{\prime}(u)$ is the generator of $F$, then for $F$ to be equivalent to (1.1) it is necessary and sufficient that there exist a sequence $\sigma$ of nonzero numbers such that $t_{p}^{\prime}(u)=t_{p}\left(u / \sigma_{p}\right)$ for $p \geqq 1$. If $\sigma$ is such a sequence, then $F$ is the continued fraction

$$
f_{1}+\frac{\sigma_{1} a_{1}}{\sigma_{1} b_{1}-\frac{\sigma_{1} \sigma_{2} a_{2}}{\sigma_{2} b_{2}-\frac{\sigma_{2} \sigma_{3} a_{3}}{\sigma_{3} b_{3}-\cdots}}} .
$$

Theorem 4.1. For (1.1) to be equivalent to a positive definite continued fraction, it is necessary and sufficient that there exist a sequence $R$ in $B_{f}$ such that if $p \geqq 1$, then $t_{p}^{-1}\left(R_{p}\right)$ is a closed half-plane; i.e., if $p \geqq 1$, then $f_{p}$ is a boundary point of $R_{p}$.

Proof. Let $t^{-1}(R)$ be a sequence of closed half-planes. Then there exist a sequence $\sigma$ of nonzero numbers and a sequence $k$ of real numbers such that if $p \geqq 1$, then $t_{p}^{-1}\left(R_{p}\right)$ is defined by $R\left(\sigma_{p} u\right) \leqq k_{p}$. We show first that for $R$ to be in $B_{f}$ it is necessary and sufficient that

(i) $0 \leqq k_{1}<R\left(\sigma_{1} b_{1}\right)$ and $0 \leqq k_{p} \leqq R\left(\sigma_{p} b_{p}\right)$ for $p>1$, and

(ii) $R\left(\sigma_{p} \sigma_{p+1} a_{p+1}\right)+\left|\sigma_{p} \sigma_{p+1} a_{p+1}\right| \leqq 2 k_{p} R\left(\sigma_{p+1} b_{p+1}-k_{p+1}\right)$ for $p \geqq 1$.

\footnotetext{
${ }^{2}$ Numbers in brackets refer to the bibliography at the end of the paper.

${ }^{8}$ This is an adaptation to (1.1) of the definition on pp. 67-71 of [3], where it is assumed that $g_{1} I\left(b_{1}\right)>0$; e.g., in formula (17.3) of [3].
} 
For $R_{1}$ to be a circle plus its interior, it is necessary and sufficient that the point $t_{1}^{-1}(\infty)=b_{1}$ be exterior to $t_{1}^{-1}\left(R_{1}\right)$; i.e., that $R\left(\sigma_{1} b_{1}\right)>k_{1}$. If $p \geqq 1$, then $\infty$ is a boundary point of $t_{p}^{-1}\left(R_{p}\right)$, and $f_{p}=t_{p}(\infty)$ is a boundary point of $R_{p}$; similarly, $f_{p+1}$ is a boundary point of $R_{p+1}$. If $R_{p} \supset R_{p+1}$, then the point $t_{p}^{-1}\left(f_{p+1}\right)=0$ is in $t_{p}^{-1}\left(R_{p}\right)$, or $0 \leqq k_{p}$; moreover, the point $t_{p+1}^{-1}\left(f_{p}\right)=b_{p+1}$ is not an interior point of $t_{p+1}^{-1}\left(R_{p+1}\right)$, or $R\left(\sigma_{p+1} b_{p+1}\right) \geqq k_{p+1}$. Hence for $t^{-1}(R)$ to be in $B_{f}$, the conditions (i) of (4.2) are necessary. by

Suppose that (i) of (4.2) holds. Then for $p \geqq 1, t_{p}^{-1}\left(R_{p+1}\right)$ is defined

$$
\begin{aligned}
& R\left(\bar{\sigma}_{p+1} \bar{a}_{p+1} u\right) \geqq 0 \text { if } R\left(\sigma_{p+1} b_{p+1}\right)=k_{p+1}, \text { or } \\
& \left|u-\frac{\sigma_{p+1} a_{p+1}}{2 R\left(\sigma_{p+1} b_{p+1}-k_{p+1}\right)}\right| \leqq \frac{\left|\sigma_{p+1} a_{p+1}\right|}{2 R\left(\sigma_{p+1} b_{p+1}-k_{p+1}\right)} \\
& \text { if } R\left(\sigma_{p+1} b_{p+1}\right)>k_{p+1} .
\end{aligned}
$$

Hence if (i) of (4.2) holds, then (ii) is a condition necessary and sufficient for the relations $R_{p} \supset R_{p+1}$ to hold for $p \geqq 1$. We conclude that $t^{-1}(R)$ is in $B_{f}$ if and only if (4.2) holds.

If for $p \geqq 1$ we take $\sigma_{p}=-i$ and $k_{p}=\left(1-g_{p}\right) R\left(-i b_{p}\right)$, where $g_{p}=1$ if $k_{p}=0$, the theorem now follows from (4.1) and Remark 4.1. This completes the proof.

REMARK 4.2. By Theorem 4.1, a bounded increasing infinite sequence of real numbers is the sequence of approximants of a positive definite continued fraction. More generally, if $x$ is a sequence of numbers, if $x_{p} \neq x_{p+1}$ for $p \geqq 1$, and if there exists a number $c$ such that $\left|x_{p}-c\right| \geqq\left|x_{p+1}-c\right|$ for $p \geqq 1$, then $x$ is the sequence of approximants of a positive definite continued fraction.

THEOREM 4.2. If (1.1) converges, then there exists a positive definite continued fraction whose sequence of approximants is a subsequence of $f$.

Proof. Let $c$ be the number such that $f_{p} \rightarrow c$ as $p \rightarrow \infty$. Then there exists an infinite subsequence, $x$, of $f$ such that if $p \geqq 1$, then $x_{p} \neq \infty$ and $\left|x_{p}-c\right|>\left|x_{p+1}-c\right|$. By Remark 4.2, $x$ is the sequence of approximants of a positive definite continued fraction. This completes the proof.

5. Absolute convergence of positive definite continued fractions. Throughout this section we suppose that (1.1) is equivalent to a positive definite continued fraction, and that $k$ is a sequence of real numbers such that $R$ is in $B_{f}$, where, for $p \geqq 1, t_{p}^{-1}\left(R_{p}\right)$ is the closed halfplane $R(u) \leqq k_{p}$. The conditions (4.2) hold, therefore, with $\sigma_{p}=1$, and $t_{p}^{-1}\left(R_{p+1}\right)$ is the region defined by $(4.3)$, for $p \geqq 1$. 
THEOREM 5.1. If there exist a positive integer $n$ and a positive number $M$ such that $\left|a_{p+1}\right| \leqq M k_{p} R\left(b_{p+1}-k_{p+1}\right)$ for $p \geqq n$, then (1.1) converges absolutely.

Proof. Since, by hypothesis, (1.1) is equivalent to a positive definite continued fraction, its sequence of approximants is bounded; and by (2.4), $h_{p} \neq \infty$ and $h_{p} \neq 0$ for $p \geqq 1$. Moreover, if $p \geqq n$, then $t_{p}^{-1}\left(R_{p+1}\right)$ is a circle plus its interior; let $v_{p}$ be the point of $t_{p}^{-1}\left(R_{p+1}\right)$ farthest from $h_{p}$. By (2.5),

$$
\left|\frac{f_{p+1}-f_{p}}{t_{p}\left(v_{p}\right)-f_{p}}\right|=\left|\frac{h_{p}-v_{p}}{h_{p}}\right| \leqq 1+\left|\frac{v_{p}}{h_{p}}\right| .
$$

Since the origin is a boundary point of $t_{p}^{-1}\left(R_{p+1}\right),\left|v_{p}\right|$ is less than or equal to the diameter of $t_{p}^{-1}\left(R_{p+1}\right)$, or $\left|v_{p}\right| \leqq\left|a_{p+1}\right| / R\left(b_{p+1}-k_{p+1}\right)$; hence $\left|v_{p}\right|<M k_{p}$. Since $R_{p}$ is a circle plus its interior, $h_{p}$ is not in the closed half-plane $R(u) \leqq k_{p}$; so $\left|h_{p}\right| \geqq R\left(h_{p}\right)>k_{p}$. Finally, by (2.5), $t_{p}\left(v_{p}\right)$ is the point of $R_{p+1}$ nearest $f_{p}$; so $\left|t_{p}\left(v_{p}\right)-f_{p}\right| \leqq 2\left(r_{p}-r_{p+1}\right)$, where for $p \geqq 1, r_{p}$ is the radius of $R_{p}$. We now conclude that if $p \geqq n$, then $\left|f_{p+1}-f_{p}\right|<2(1+M)\left(r_{p}-r_{p+1}\right)$. Since $\sum_{p=n}^{\infty}\left(r_{p}-r_{p+1}\right)$ is a convergent positive-term series, (1.1) converges absolutely. This completes the proof.

COROLlARY 5.1a. If there exist a sequence $g$ and a positive number $M$ such that, for $p \geqq 1$,

(i) $0<g_{p}<1$,

(ii) $\left|c_{p}\right|-R\left(c_{p}\right) \leqq 2\left(1-g_{p}\right) g_{p+1}$, and

(iii) $\left|c_{p}\right|<M\left(1-g_{p}\right) g_{p+1}$,

then the continued fraction (3.4) converges absolutely.

REMARK 5.1. The above corollary is a true generalization of the convergence condition $\left|c_{p}\right| \leqq\left(1-g_{p}\right) g_{p+1}, p \geqq 1$, of Pringsheim [4]; compare it with the condition $\left|c_{p}\right|-R\left(c_{p}\right) \leqq 2 r\left(1-g_{p}\right) g_{p+1}$, where $0<r<1, p \geqq 1$, on pp. $142-143$ of [3].

REMARK 5.2. It should be noted that in Theorem 5.1 and its corollary we do not conclude that the common part of $R_{1}, R_{2}, R_{3}, \cdots$ is a point. Actually there exists an absolutely convergent positive definite continued fraction which has the property that if $t^{-1}(R)$ is a sequence of closed half-planes such that $R$ is in $B_{f}$, then the common part of $R_{1}, R_{2}, R_{3}, \ldots$ is a circle plus its interior. We give the following example. Let $s$ be a decreasing sequence of positive numbers such that $\sum_{p-1}^{\infty} s_{p}$ converges. For $p \geqq 1$, let each of $R_{3 p-2}, R_{3 p-1}$, and $R_{3 p}$ be the region defined by $\left|u-\left(s_{p}-1\right)\right| \leqq 1+s_{p}$, and let $f_{3 p-2}, f_{3 p-1}$, 
and $f_{3 p}$ be boundary points of $R_{3 p}$ such that $\arg f_{3 p-2}=0, \arg f_{3 p-1}=1$, and $\arg f_{3 p}=-1$. Then $f$ is the sequence of approximants of an absolutely convergent positive definite continued fraction. If $R^{\prime}$ is a sequence in $B_{f}$ such that $f_{p}$ is a boundary point of $R_{p}^{\prime}$ for $p \geqq 1$, then $R_{3 p-2}^{\prime} \supset R_{3 p-2}$ for $p \geqq 1$, and hence the common part of $R_{1}^{\prime}, R_{2}^{\prime}, R_{3}^{\prime}, \ldots$ is a circle plus its interior.

THEOREM 5.2. Let $e_{p}=\left|a_{p+1}\right| /\left[2 k_{p} R\left(b_{p+1}-k_{p+1}\right)-R\left(a_{p+1}\right)\right]$ for $p \geqq 1$. If $\sum_{p=1}^{\infty}\left(1-e_{p}\right)$ diverges, then (1.1) converges. If there exists a sequence $d$ of positive numbers such that $e_{p}\left(2+2 d_{p+1}-d_{p}\right) \leqq d_{p}$ for $p \geqq 1$, then (1.1) converges absolutely.

Proof. A. We show first that $r_{p+1} / r_{p} \leqq 2 e_{p} /\left(1+e_{p}\right)$ for $p \geqq 1$, where $r_{p}$ is the radius of $R_{p}$. If $R\left(b_{p+1}\right)=k_{p+1}$, then by (4.2) $a_{p+1}<0$ and hence $e_{p}=1$, so that the relation $r_{p+1} / r_{p} \leqq 2 e_{p} /\left(1+e_{p}\right)$ holds. If $R\left(b_{p+1}\right)>k_{p+1}$, then $t_{p}^{-1}\left(R_{p+1}\right)$ is a circle plus its interior; let $v_{p}$ be the point of $t_{p}^{-1}\left(R_{p+1}\right)$ farthest from $h_{p}$, and let $w_{p}$ be the point of $t_{p}^{-1}\left(R_{p+1}\right)$ nearest $h_{p}$. By (2.5), $t_{p}\left(v_{p}\right)$ is the point of $R_{p+1}$ nearest $f_{p}$, and $t_{p}\left(w_{p}\right)$ is the point of $R_{p+1}$ farthest from $f_{p}$; so $2 r_{p+1}=\left|t_{p}\left(v_{p}\right)-t_{p}\left(w_{p}\right)\right|$ and $2 r_{p} \geqq\left|t_{p}\left(w_{p}\right)-f_{p}\right|$. But by (2.5), $\left[t_{p}\left(v_{p}\right)-t_{p}\left(w_{p}\right)\right] /\left[t_{p}\left(w_{p}\right)-f_{p}\right]=\left|\left(v_{p}-w_{p}\right) /\left(h_{p}-v_{p}\right)\right|$; hence $r_{p+1} / r_{p} \leqq\left|\left(v_{p}-w_{p}\right) /\left(h_{p}-v_{p}\right)\right|$. Since the diameter of $t_{p}^{-1}\left(R_{p+1}\right)$ is $\left|v_{p}-w_{p}\right|=\left|a_{p+1}\right| / R\left(b_{p+1}-k_{p+1}\right)$, and since the distance from $h_{p}$ to $v_{p}$ is $\left|h_{p}-v_{p}\right|>k_{p}+\left[\left|a_{p+1}\right|-R\left(a_{p+1}\right)\right] / 2 R\left(b_{p+1}-k_{p+1}\right)$, it follows that

$$
\frac{r_{p+1}}{r_{p}} \leqq \frac{2\left|a_{p+1}\right|}{2 k_{p} R\left(b_{p+1}-k_{p+1}\right)-R\left(a_{p+1}\right)+\left|a_{p+1}\right|}=\frac{2 e_{p}}{1+e_{p}} .
$$

B. Suppose that $\sum_{p=1}^{\infty}\left(1-e_{p}\right)$ diverges. Now by definition and by (4.2), $0<e_{p} \leqq 1$; so $\sum_{p=1}^{\infty}\left(1-e_{p}\right) /\left(1+e_{p}\right)$ diverges. But $1-r_{p+1} / r_{p}$ $\geqq 1-2 e_{p} /\left(1+e_{p}\right)=\left(1-e_{p}\right) /\left(1+e_{p}\right)$. Hence if for $p \geqq 1, s_{p}=1$ $-r_{p+1} / r_{p}$, then $\sum_{p=1}^{\infty} s_{p}$ is a divergent series whose terms are nonnegative real numbers. Since $r_{p+1}=r_{1}\left(1-s_{1}\right)\left(1-s_{2}\right) \cdots\left(1-s_{p}\right)$, it follows that $r_{p} \rightarrow 0$ as $p \rightarrow \infty$, and consequently (1.1) converges.

C. Suppose that there exists a sequence $d$ of positive numbers such that $e_{p}\left(2+2 d_{p+1}-d_{p}\right) \leqq d_{p}$ for $p \geqq 1$. Then for $p \geqq 1, r_{p+1} / r_{p}$ $\leqq 2 e_{p} /\left(1+e_{p}\right) \leqq d_{p} /\left(1+d_{p+1}\right)$; and by Lemma 3.2a, $\sum_{p+1}^{\infty} r_{p}$ converges. Since $\left|f_{p+1}-f_{p}\right| \leqq 2 r_{p},(1.1)$ converges absolutely. This completes the proof of the theorem.

EXAMPLE 5.1. Let $s$ be a positive number greater than 4 . If $0<c_{p}$ $\leqq p / s$ for $p \geqq 1$, then the continued fraction (3.4) converges absolutely. This can be seen by taking $k_{p}=1 / 2$ and $d_{p}=4 p /(s-4)$ in Theorem 5.2. In Corollary $6.1 \mathrm{a}$, p. 380 , of [1], it was required that an infinite subsequence of $c$ be bounded. 
REMARK 5.3. If $c_{p}=p(p+x) /(1+x)^{2}$ for $p \geqq 1$, it can be shown that (3.4) converges absolutely for $x>0$; it was shown on p. 379 of [1] that if $x=0$, then (3.4) converges but does not converge absolutely.

\section{BIBLIOGRAPHY}

1. R. E. Lane, and H. S. Wall, Continued fractions with absolutely convergent even and odd parts, Trans. Amer. Math. Soc. vol. 67 (1949) pp. 368-380.

2. W. T. Scott and H. S. Wall, A convergence theorem for continued fractions, Trans. Amer. Math. Soc. vol. 47 (1940) pp. 155-172.

3. H. S. Wall, Analytic theory of continued fractions, New York, 1948.

4. A. Pringsheim, Über die Konvergenz unendlicher Ketlenbriiche, Sitzungsberichte der Mathematisch-Naturwissenschaftlichen Klasse der Bayerischen Akademie der Wissenschaften zu München vol. 28 (1898) pp. 295-324.

UNIVERSITY OF TEXAS 